\title{
Stereological Recovery of Five-Parameter Grain Boundary Character Distributions from EBSD Maps
}

\author{
C.-S. Kim, J. Gruber, and G.S. Rohrer
}

Department of Materials Science and Engineering, Carnegie Mellon University, 5000 Forbes Avenue, Pittsburgh, PA 15213-3890, USA

The grain boundary distribution, $\square(\square g, \mathbf{n})$, is defined as the relative areas of grain boundaries with misorientation, $\square g$, and boundary plane normal, $\mathbf{n}$, in units of multiples of a random distribution (MRD). Each grain boundary therefore has five parameters, three that describe the misorientation and two that describe the boundary plane normal [1]. Two stereological methods have recently been described for measuring the grain boundary character distribution from electron backscattered diffraction (EBSD) maps $[2,3]$. The purpose of the current paper is to describe a new method, based on reference [3], that more accurately reconstructs the grain boundary character distribution.

EBSD maps from single section planes can be used to specify four of the five grain boundary parameters; the only unknown parameter is the inclination angle between the observation plane and the grain boundary plane. To find the distribution of grain boundary plane orientations, we make observations of many crystal pairs that have indistinguishable misorientations, but are oriented differently with respect to the section plane. Knowing that the true boundary plane (n) must be in the zone of the observed boundary line segment $(\boldsymbol{l})$ such that $\mathbf{n} \boldsymbol{\bullet} \boldsymbol{l}=0$, the grain boundary trace associated with each bicrystal generates a set of possible boundary planes in the bicrystal reference frame. The probability that each set contains the true boundary plane is one, and the probability that any arbitrary plane is included in the set is less than one. Therefore, after many observations of the same type of bicrystal, true boundary planes make up a relatively larger fraction of the accumulated data than the arbitrary planes, and can therefore be identified as peaks in the distribution.

To quantify the areal distribution of the boundary planes, we have to account for the fact that the observed area for a certain plane is the sum of its true area and erroneous contributions from other grain boundary types in the same zone. In ref. 3, a mean field approximation was used to subtract the erroneous contributions. While this approximate method is acceptable for grain boundary character distributions with weak anisotropy, the errors are more significant when the grain boundary plane texture is large. We have implemented a new scheme that separately considers the erroneous contributions from all other grain boundary types and produces a more accurate result (see Figs. 1 and 2). Simulation results will be presented that demonstrate the reliability of the method for samples with different crystal symmetries and varying degrees of anisotropy. Experimental results from cubic, tetragonal, hexagonal, and trigonal materials will also be presented.

\section{References}

[1] G.S. Rohrer, D.M. Saylor, B.S. El-Dasher, B.L. Adams, A.D. Rollett, and P. Wynblatt, Z. Metall., 95 (2004) 214.

[2] R.J. Larsen and B.L. Adams, Metall. Mater. Trans., 35A (2004) 1991.

[3] D.M. Saylor, B.L. Adams, B.S. El Dasher, G.S. Rohrer, Metall. Mater. Trans., 35A (2004) 1981.

[4] Supported by the MRSEC program of the NSF under award number DMR-0079996. 

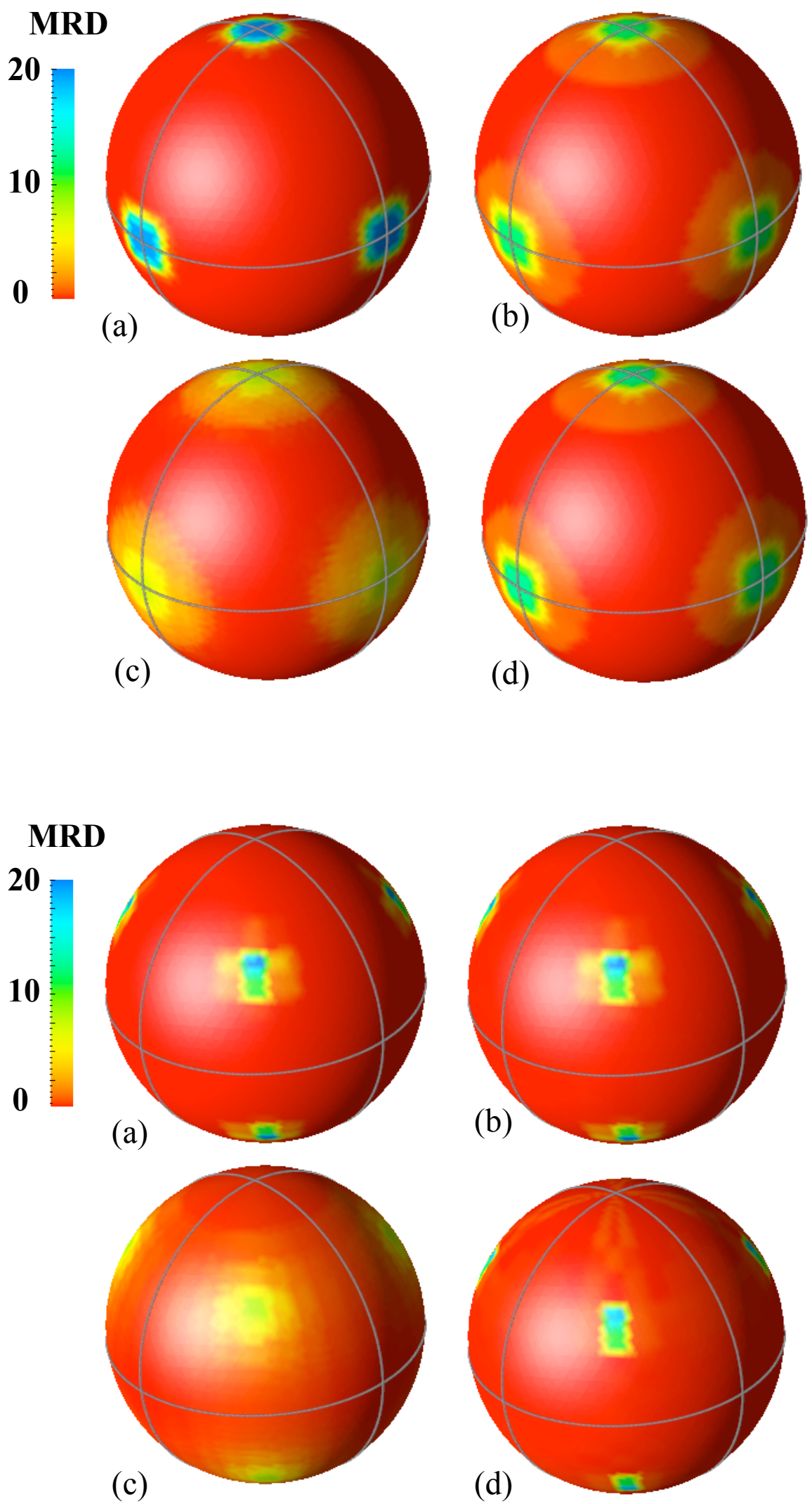

Figure 1. The distribution of grain boundary planes, independent of the lattice misorientation. The distribution is plotted on the surface of a sphere in the crystal reference frame. The gray lines intersect at $\{100\}$ poles. (a) Model cubic distribution, with a maximum value of 19.6 at $\{100\}$. (b) The distribution of 50,000 randomly sampled grain boundary traces. Here the maximum value is 11.9 .

(c) Reconstructed distribution using method in [3], Here the maximum value is 7.3 . (d) Reconstructed distribution using the new method. Here, the maximum value is 12.1 , which is very close to the sampled distribution.

Figure 2. The distribution of grain boundary planes, as above, but for tetragonal symmetry. (a) In the model distribution, the maximum value is 20.7 at $\{111\}$. (b) The distribution of 50,000 randomly sampled grain boundary traces. Here the maximum value is 20.8 . (c) Reconstructed distribution using method in [3], Here the maximum value is 8.1 .

(d) Reconstructed distribution using the new method. Here the maximum value is 18.8 , which is very close to the observed or sampled distribution. 